\title{
Serological Survey of Leptospirosis among Poultry Farm and Slaughterhouse Workers and its Co-relation with the Risk Factors
}

\author{
Yogendra Mevada $^{1 *}$, Rajendra Palkhade ${ }^{2 *}$, Utpal Kumar Chattopadhyay ${ }^{1}$, \\ Amit Kanani $^{3}$, Atul Raj ${ }^{4}$, Amit Banik ${ }^{4}$, Sayan Bhattacharyya ${ }^{4}$, Subhrajit Sarkar ${ }^{5}$, \\ Nisha Gupta ${ }^{3}$ and Jitendra Parmar ${ }^{2}$ \\ ${ }^{1} \mathrm{MVPH},{ }^{4}$ Department of Microbiology, ${ }^{5}$ Department of Statistics, AIIH \& PH, Kolkata, India \\ ${ }^{2}$ ICMR-NIOH, ${ }^{3}$ Department of Animal Husbandry, Ahmedabad, India \\ *Corresponding author
}

\begin{tabular}{|l|}
\hline K e y w o r d s \\
Leptospirosis, \\
ELISA, Occupation, \\
Antibodies, Risk \\
factors
\end{tabular}

Keywords

Leptospirosis, ELISA, Occupation, Antibodies, Risk

Accepted:

26 August 2020

10 September 2020

\section{A B S T R A C T}

Leptospirosis is an acute, bacterial, anthropozoonotic disease. Some occupations are highrisk group occupations for Leptospirosis e.g. field veterinarian, animal handler, dairy workers, slaughterhouse workers, rice field workers, sugar-cane field workers, sewage workers, and laboratory personnel. Poultry is known to be resistant to Leptospira spp. infection but the feed stuff available at poultry farms which are mostly located in the field attracts wild rodents. So it is important to study the prevalence of Leptospira spp. among poultry farm workers and slaughterhouse workers and its correlation with their occupational practices. To determine the prevalence of Leptospira spp. among poultry farm \& slaughterhouse workers and its correlation with the occupational practices of them. To check the presence of Leptospiral antibodies in carrier and reservoir animals, present in the working environment of poultry farms and slaughterhouses. A cross-sectional survey was conducted in 2017 at poultry farms of Navsari district and slaughterhouse of two cities of Gujarat viz., Ahmedabad and Surat. A total of 108 asymptomatic workers, 50 poultry farm and 58 slaughterhouse workers were enrolled with their written consent; a blood sample was collected and subjected to Leptospira spp. IgG ELISA. Information regarding occupational practices was collected using predesigned, semi-structured validate proforma. To check the presence of Leptospiral antibodies a total of 83 animals' blood samples collected from poultry farms $(n=28)$ and slaughterhouses $(n=55)$. The collected data were co-related and analysed using SPSS version 25 . The current study showed that the prevalence of Leptospira spp. among the poultry farm workers of Navsari district was $4 \%$ $(\mathrm{n}=50)$. The study showed that the prevalence of Leptospira spp. among the slaughterhouse workers was $12.06 \%(\mathrm{n}=58)$. Leptospiral antibodies reported in $3.57 \%$ $(\mathrm{n}=28)$ and $1.81 \%(\mathrm{n}=55)$ of carrier and reservoir animals present at poultry farms and slaughterhouses respectively.

\section{Introduction}

The Leptospirosis is an acute, bacterial, anthropozoonotic disease. It's an occupational hazard. Leptospires are transmitted directly or indirectly from animals to humans. Human to human transmission occurs very rarely. Pathogenic leptospires are maintained in 
nature in the renal tubules and genital tracts of certain animals. Leptospires spread through the urine of certain animals and contaminate the environment. Leptospirosis can cause mild-flu like symptoms such as fever, headache, chills, and muscle pain in the majority of cases, but in some cases, more severe infection can occur and it may cause life-threatening problems like organ failure and internal bleeding. This severe form is also known as Weil's disease. The diagnosis of Leptospirosis in humans is difficult in the case of mild symptoms, but easy when the infection causes serious health problems. Leptospira spp. causes infection primarily among animals, whereas human beings are accidental hosts. It causes infection in man or animals when such susceptible host comes in contact (abraded skin) with water which is contaminated by infected animal urine.

The infection may also be transmitted through direct contact with urine or tissues of infected animals, or by inhalation of aerosols of contaminated fluids, as condition mostly occurs in abattoirs. Ingestion of foods contaminated with the urine of infected rats is also an occasional route of infection. Leptospirosis is highly prevalent in some of the states and union territories of India like Andaman and Nicobar Islands, Kerala, Tamilnadu Gujarat, Maharashtra, Andhra Pradesh, Assam, Goa, Delhi, Karnataka, Orissa, Puducherry, and Uttar Pradesh. In Gujarat it's mainly reported from South Gujarat (Surat, Navsari, Valsad, Tapi, and Dang districts) [1]. South Gujarat is an endemic area for Leptospirosis. Most areas of the districts of South Gujarat are used for sugarcane and paddy farming and are rich in natural flora with plenty of marshy lands and small ponds/water logging areas with almost neutral $\mathrm{pH}$, required humidity, and temperature which are optimum for growth/survival of leptospires and perpetuation in the environment [2].
Leptospirosis cases were first seen in Gujarat in 1994, in the Chikhli block of the old Valsad district. Since then cases of Leptospirosis were continuously reported from Surat, Tapi, Navsari and Valsad districts of South Gujarat. Sporadic cases of Leptospirosis are being reported throughout the year in the country, but Leptospirosis epidemic reported mostly during the monsoon season (August - December) and lasting for few months [3]. Due to the unavailability of appropriate laboratory diagnostic facilities in most parts of the country, lack of awareness of the disease among the public health workers, and inadequate epidemiological data, Leptospirosis has been under-reported and under-diagnosed in India. [4]. High-risk occupational environments provide favourable conditions for the prolonged survival of Leptospira spp. such occupations are, e.g. field veterinarian, animal handler, dairy workers, slaughterhouse workers, rice field, sugar-cane field workers, sewage workers, laboratory personnel, poultry farm workers, etc. It is also reported in people who participate in leisurely and recreational activities like swimming, sailing, canoeing, rafting, caving, canyoneering, fishing, etc. Poultry is known to be resistant to Leptospira spp. infection but the feed available at poultry farms attract wild rodents and make the poultry farm workers prone to infection. So, it is important to study the prevalence of Leptospira spp. among poultry farmworkers. Although slaughterhouse workers had known risk of exposure to many zoonotic infections due to virtue of their occupation and very few studies were conducted pertaining to this occupation in the Gujarat state, so, it is also important to study the prevalence of Leptospira spp. among slaughterhouse workers. Objectives: To determine the prevalence of Leptospira spp. among poultry farm \& slaughterhouse workers and its correlation with the occupational practices. To check the presence of Leptospiral 
antibodies in carrier and reservoir animals, present in the working environment of poultry farms and slaughterhouses.

\section{Materials and Methods}

\section{Study design, population, and area}

An occupation based cross-sectional survey was conducted from July-2017 to Jan-2018 at poultry farms of Navsari district and slaughterhouse of two cities of Gujarat viz: Ahmedabad \& Surat. Involved Study participants were from eight (8) different poultry farms from 4 blocks of Navsari district and two (2) slaughterhouses (Ahmedabad \& Surat) of Gujarat state.

\section{Sampling}

We did two types of sampling in this study. 1. Purposive sampling for selection of the district, block, and slaughterhouses. 2. Simple random sampling for selection of poultry farm, farm workers, slaughterhouse workers, animals present at enrolled poultry farms and animals slaughter at the slaughterhouse. We kept a minimum target of 50 study participants from each workgroup. As Leptospirosis first reported in chikli block of the old Valsad district of South Gujarat [3], which is now in Navsari district, so, we randomly targeted eight (8) poultry farms of four (4) different blocks of Navasari district and randomly enrolled 50 asymptomatic poultry farm workers.

The slaughterhouses of Surat and Ahmedabad selected by purposive sampling, because they are two of the main slaughter houses in the Gujarat state. We randomly selected 58 asymptomatic slaughterhouse workers, 40 from Surat and 18 from Ahmedabad. The animals present at poultry farms $(n=23)$ and slaughtered in slaughterhouses $(n=55)$ were selected randomly.

\section{Tools and technique}

\section{Human samples}

After taking written consent; a blood sample of the enrolled study population was collected and transferred to the laboratory. The serum was separated by centrifuging at 1500 RPM for 15 minutes and subjected to Leptospira spp. IgG ELISA (Nova Tec-NovaLisa LeptospiraIg G ELISA).

\section{Animal samples}

A Total of 83 animals' blood samples, 28 present at a poultry farm, and 55 slaughtered in slaughterhouses, were collected. Rats were collected from a poultry farm in food bait traps (with Sherman-type hook); the blood was collected from the tail vein of a rat. After the collection of blood, a rat was released at their respective place of collection. The serum was separated by centrifuging at 1500 RPM for 15 minutes and subjected to Indirect ELISA (Genomix Leptospira Indirect ELISA).

\section{Data collection}

Data were collected by interviewing study participants for Information regarding demography, workplace environment, and occupational practices, using predesigned, semi-structured validated proforma.

\section{Statistical analyses}

The collected data were entered into Microsoft Excel and analyzed using SPSS version 25. Workers' socio-economic (S-E) class was calculated using the Modified kappuswami scale proposed update 2017 [5]. The correlation between the data and ELIZA results was established by the Chi-square test and Fisher exact ratio. $\mathrm{P} \leq 0.05$ was considered as statistically significant. 


\section{Ethical consideration}

The Human Ethical clearance was obtained from the Institutional Human Ethical committee, ICMR-NIOH, Agenda No. 3.8, Dt. June 19, 2017. (Project title: Seroprevalence of Leptospirosis among high-risk group occupations).

\section{Results and Discussion}

The prevalence of Leptospira spp. among poultry farm and slaughterhouse workers was found to be $4.0 \%$ and $12.06 \%$ respectively. The demographic distribution of the study population is mentioned in table 1 .

The mean age of poultry farm and slaughterhouse workers was found to be 33.48 \pm 11.22 and $33.78 \pm 11.43$ respectively. The association between ELISA results and demographic distribution, environmental, and behavioral risk factors is mentioned in table 2 , 3,4 .

At the time of comparison between Human Leptospira spp. IgG ELISA results and demographic characteristics, we found that among 2 sero-positive poultry farm workers, 1 was male and 1 was female. According to the age group, the majority of sero-positive poultry farm workers were from 25 to 44 years.

The education-wise majority of sero-positive poultry farm workers had higher secondary and above education. According to family size all sero-positive poultry farm workers had 3 to 5 members in one family.

According to socio-economic class one of the sero-positive poultry farm worker belonged to the lower middle and another belonged to the upper-lower S-E class. According to work experience all sero-positive poultry farm workers had work experience of 5-19 years. Whereas in slaughterhouse workers, we found that all sero-positive slaughterhouse workers were male.

The ages group-wise, among 7 sero-positive slaughterhouse workers 4 belonged to 25-44 years and 3 belonged to 45 and above years.

The education-wise majority of sero-positive slaughterhouse workers had higher secondary and above education. According to family size all sero-positive slaughterhouse workers had 3 to 5 members in one family.

The socio-economic class-wise, among 7 sero-positive slaughterhouse workers 4 belonged to upper-middle and 3 belonged to lower-middle S-E class. According to work experience the majority of sero-positive slaughterhouse workers had work experience of 20 years and above.

When we co-related Human Leptospira spp. IgG ELISA result with environmental risk factors, we found that 2 sero-positive poultry farm workers were living in the field areas.

All the sero-positive poultry farm workers had presence of livestock, mice/rats at home and workplaces and also had the presence of rats/mice in a drain of workplace.

Whereas, one of the sero-positive poultry farm workers had history of livestock in the home. In slaughterhouse workers, we found that the majority of sero-positive slaughterhouse workers were living nearby market area and had the history of presence of $\mathrm{rat} /$ mice in drains at workplace.

One of the sero-positive slaughterhouse workers had livestock in the home. All seropositive slaughterhouse workers had history of mice/rats at the workplace 
Table.1 Demographic distribution of study population

\begin{tabular}{|c|c|c|}
\hline Variable & \multicolumn{2}{|c|}{$\mathrm{n}(\%)$} \\
\hline \multicolumn{3}{|l|}{ Profession } \\
\hline Poultry farm worker & \multicolumn{2}{|c|}{$41(82)$} \\
\hline Poultry farm worker + Farmer & \multicolumn{2}{|c|}{$04(08)$} \\
\hline Poultry farm worker + Owner + Manager & \multicolumn{2}{|c|}{$05(6.25)$} \\
\hline Total (group of poultry farm workers) & \multicolumn{2}{|c|}{$50(100)$} \\
\hline Large animal butcher & \multicolumn{2}{|c|}{$20(34.48)$} \\
\hline Large animal helper & \multicolumn{2}{|c|}{$04(6.89)$} \\
\hline Small animal butcher & \multicolumn{2}{|c|}{$27(46.55)$} \\
\hline Small animal helper & \multicolumn{2}{|c|}{$02(3.44)$} \\
\hline Slaughterhouse sweeper & \multicolumn{2}{|c|}{$05(8.62)$} \\
\hline Total (group of slaughterhouse workers) & \multicolumn{2}{|c|}{$58(100)$} \\
\hline Gender & $\begin{array}{c}\text { Poultry } \\
\text { Farm }\end{array}$ & Slaughterhouse \\
\hline Male & $26(52)$ & $58(100)$ \\
\hline Female & $24(48)$ & 00 \\
\hline \multicolumn{3}{|l|}{ Age group (Years) } \\
\hline 18-24 & $12(24)$ & $08(13.7)$ \\
\hline $25-44$ & $32(64)$ & $39(67.2)$ \\
\hline $45 \&$ above & $06(12)$ & $11(18.9)$ \\
\hline \multicolumn{3}{|l|}{ Education } \\
\hline Illiterate & $18(36)$ & $07(12.06)$ \\
\hline Primary School & $19(38)$ & $21(36.20)$ \\
\hline Secondary school & $09(18)$ & $28(48.27)$ \\
\hline Higher Secondary school and above & $04(08)$ & $02(3.443)$ \\
\hline \multicolumn{3}{|l|}{ Family size } \\
\hline$\leq 2$ & $07(14)$ & $02(3.44)$ \\
\hline 3 to 5 & $40(80)$ & $46(79.31)$ \\
\hline$\geq 6$ & $03(06)$ & $10(17.24)$ \\
\hline \multicolumn{3}{|l|}{ S-E Class } \\
\hline Upper middle & $08(16)$ & $42(72.41)$ \\
\hline Lower middle & $11(22)$ & $14(24.13)$ \\
\hline Upper lower & $31(62)$ & $02(3.44)$ \\
\hline \multicolumn{3}{|l|}{ Work Experience (Years) } \\
\hline $1-4$ & $27(54)$ & $18(31.3)$ \\
\hline $5-19$ & $20(40)$ & $26(44.8)$ \\
\hline 20 years and above & $03(06)$ & $14(24.1)$ \\
\hline
\end{tabular}


Table.2 Comparisons of Leptospira spp. IgG ELISA result with demographic distributions

\begin{tabular}{|c|c|c|c|c|c|c|}
\hline \multirow{3}{*}{$\begin{array}{l}\text { Variable } \\
\text { Sex }\end{array}$} & \multicolumn{6}{|c|}{ Leptospira spp.IgG ELISA Result } \\
\hline & \multicolumn{2}{|c|}{ Positive (n) } & \multicolumn{2}{|c|}{ Equivocal (n) } & \multicolumn{2}{|c|}{ Negative $(\mathrm{n})$} \\
\hline & $\begin{array}{c}\text { Poultry } \\
\text { farm } \\
\text { worker }\end{array}$ & $\begin{array}{l}\text { Slaughterhouse } \\
\text { worker }\end{array}$ & $\begin{array}{c}\text { Poultry } \\
\text { farm } \\
\text { worker }\end{array}$ & $\begin{array}{l}\text { Slaughterhouse } \\
\text { worker }\end{array}$ & $\begin{array}{l}\text { Poultry } \\
\text { farm } \\
\text { worker }\end{array}$ & $\begin{array}{l}\text { Slaughterhouse } \\
\text { worker }\end{array}$ \\
\hline Male & 01 & 07 & 02 & 03 & 23 & 48 \\
\hline Female & 01 & 0 & 00 & 0 & 23 & 0 \\
\hline \multicolumn{7}{|c|}{$\begin{array}{c}\chi 2 \text { statistics for Poultry farm Workers }=1.923, \mathrm{df}=2, \mathrm{p} \text { value }=0.382 \text {, Non-Significant as } p>0.05 \\
\chi 2 \text { statistics for Slaughterhouse Workers was not compared because of constant response } \\
\text { For poultry farm worker } 4 \text { cells }(66.7 \%) \text { have expected count }<5 \text {, so Fisher's exact test }=1.795, P=0.740 \\
\text { Non-Significant as } P>0.05\end{array}$} \\
\hline \multicolumn{7}{|l|}{$\begin{array}{l}\text { Age group } \\
\text { (yrs.) }\end{array}$} \\
\hline $18-24$ & 00 & 00 & 00 & 00 & 12 & 08 \\
\hline $25-44$ & 01 & 04 & 02 & 02 & 29 & 33 \\
\hline $45 \&$ above & 01 & 03 & 00 & 01 & 05 & 07 \\
\hline \multicolumn{7}{|c|}{$\begin{array}{c}\chi 2 \text { statistics for Poultry farm Workers }=3.125, \mathrm{df}=4, \mathrm{P} \text { value }=0.537, \text { Non-Significant as } P>0.05 \\
\chi 2 \text { statistics for Slaughterhouse Workers }=5.594, \mathrm{df}=4, \mathrm{P} \text { value }=0.232, \text { Non-Significant as } P>0.05 \\
\text { For poultry farm worker } 6 \text { cells }(66.7 \%) \text { have expected count }<5, \text { so Fisher's exact test }=2.723, P=0.628 \\
\text { Non-Significant as } P>0.05\end{array}$} \\
\hline \multicolumn{7}{|c|}{ 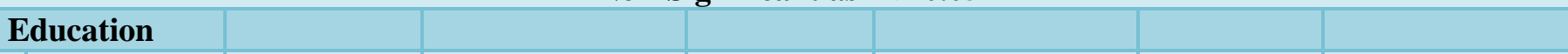 } \\
\hline Illiterate & 01 & 00 & 00 & 00 & 17 & 07 \\
\hline $\begin{array}{l}\text { Primary } \\
\text { School }\end{array}$ & 00 & 01 & 00 & 00 & 06 & 11 \\
\hline $\begin{array}{l}\text { Secondary } \\
\text { school }\end{array}$ & 00 & 02 & 00 & 00 & 02 & 08 \\
\hline $\begin{array}{l}\text { Higher } \\
\text { Secondary } \\
\text { school and } \\
\text { above }\end{array}$ & 01 & 04 & 02 & 03 & 21 & 22 \\
\hline \multicolumn{7}{|c|}{$\begin{array}{c}\chi 2 \text { statistics for Poultry farm Workers }=33.18, \mathrm{df}=10, \mathrm{P} \text { value }=0.000, \text { Significant as } \mathrm{p}<0.05 \\
\chi 2 \text { statistics for Slaughterhouse Workers }=6.053, \mathrm{df}=8, \mathrm{P} \text { value }=0.641, \text { Non-Significant as } p>0.05 \\
\text { For poultry farm worker } 15 \text { cells }(83.3 \%) \text { have expected count }<5, \text { so Fisher's exact test }=17.420, \mathrm{P} \text { value }= \\
0.52, \text { Non-Significant as } P>0.05 \\
\text { For slaughterhouse worker } 11 \text { cells }(73.3 \%) \text { have expected count }<5 \text {, so Fisher's exact test }=4.879, \text { P value }= \\
0.833, \text { Non-Significant as } P>0.05\end{array}$} \\
\hline \multicolumn{7}{|c|}{ 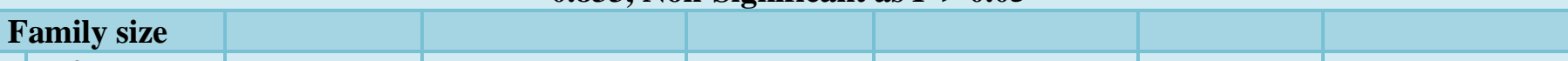 } \\
\hline$\leq 2$ & 00 & 01 & 00 & 00 & 07 & 01 \\
\hline 3 to 5 & 02 & 06 & 02 & 02 & 36 & 38 \\
\hline$\geq 6$ & 00 & 00 & 00 & 01 & 03 & 09 \\
\hline \multicolumn{7}{|c|}{$\begin{array}{r}\chi 2 \text { statistics for Poultry farm Workers }=1.087, \mathrm{df}=4, \mathrm{P} \text { value }=0.896, \text { Non-Significant as } \mathrm{P}>0.005 \\
\chi^{2} \text { statistics for Slaughterhouse Workers }=4.565, \mathrm{df}=4, \mathrm{P} \text { value }=0.335, \text { Non-Significant as } \mathrm{P}>0.005 \\
\text { For poultry farm worker } 7 \text { cells }(77.8 \%) \text { have expected count }<5, \text { so Fisher's exact test }=1.896, \mathrm{P} \text { value }=1.0, \\
\text { Non-Significant as } P>0.05 \\
\text { For slaughterhouse worker } 6 \text { cells }(66.7 \%) \text { have expected count }<5 \text {, so Fisher's exact test }=5.167, \mathrm{P} \text { value }= \\
0.265, \text { Non-Significant as } P>0.05\end{array}$} \\
\hline S-E class & & & & & & \\
\hline
\end{tabular}




\begin{tabular}{|c|c|c|c|c|c|c|}
\hline Upper middle & 00 & 04 & 02 & 03 & 05 & 36 \\
\hline Lower middle & 01 & 03 & 00 & 0 & 11 & 11 \\
\hline Upper lower & 01 & 0 & 00 & 0 & 30 & 01 \\
\hline \multicolumn{7}{|c|}{$\begin{array}{c}\chi 2 \text { statistics for Poultry farm Workers }=13.57, \mathrm{df}=4, \mathrm{P} \text { value }=0.09, \text { Non-Significant as } P>0.05 \\
\chi 2 \text { statistics for Slaughterhouse Workers }=2.526, \mathrm{df}=4, \mathrm{P} \text { value }=0.640, \text { Non-Significant as } P>0.05 \\
\text { For poultry farm worker } 6 \text { cells }(66.7 \%) \text { have expected count }<5, \text { so Fisher's exact test }=8.136, P \text { value }= \\
0.033 \text {, Non-Significant as } P>0.05 \\
\text { For slaughterhouse worker } 6 \text { cells }(66.7 \%) \text { have expected count }<5, \text { so Fisher's exact test }=3.950, P \text { value }= \\
0.540, \text { Non-Significant as } P>0.05\end{array}$} \\
\hline \multicolumn{7}{|c|}{ 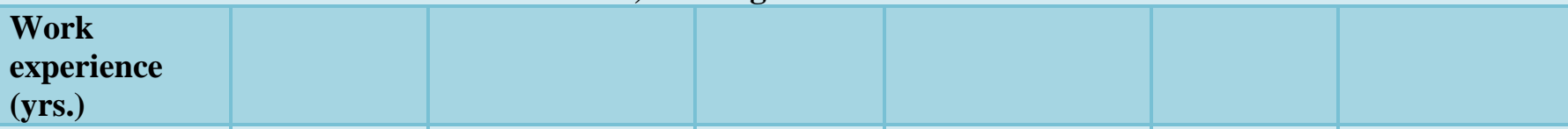 } \\
\hline $1-4$ & 0 & 00 & 0 & 01 & 27 & 17 \\
\hline $5-19$ & 02 & 02 & 02 & 01 & 16 & 23 \\
\hline $\begin{array}{l}20 \text { years and } \\
\text { above }\end{array}$ & 0 & 05 & 0 & 01 & 03 & 08 \\
\hline \multicolumn{7}{|c|}{$\begin{array}{c}\chi 2 \text { statistics for Poultry farm Workers }=6.522, \mathrm{df}=4, \mathrm{P} \text { value }=0.163, \text { Non-Significant as } P>0.05 \\
\chi 2 \text { statistics for Slaughterhouse Workers }=10.778, \mathrm{df}=4, \mathrm{P} \text { value }=0.029, \text { Non-Significant as } P>0.05 \\
\chi 2 \text { statistics for both Work groups }=15.395, \mathrm{df}=4, \mathrm{P} \text { value }=0.004, \text { Significant as } P<0.05 \\
\text { For poultry farm worker } 6 \text { cells }(77.8 \%) \text { have expected count }<5, \text { so Fisher's exact test }=6.457, \mathrm{P} \text { value }= \\
0.141, \text { Non-Significant as } P>0.05 \\
\text { For slaughterhouse worker } 6 \text { cells }(66.7 \%) \text { have expected count }<5, \text { so Fisher's exact test }=9.354, \text { P value }= \\
0.010, \text { Significant as } P<0.05\end{array}$} \\
\hline
\end{tabular}

Table.3 Comparisons of Leptospira spp. IgG ELISA result with Environmental risk factors

\begin{tabular}{|c|c|c|c|c|c|c|c|}
\hline \multirow{2}{*}{\multicolumn{2}{|c|}{ Variable }} & \multicolumn{6}{|c|}{ Leptospira spp.IgG ELISA Result } \\
\hline & & \multicolumn{2}{|c|}{ Positive (n) } & \multicolumn{2}{|c|}{ Equivocal (n) } & \multicolumn{2}{|c|}{ Negative (n) } \\
\hline \multicolumn{2}{|c|}{ Topography } & $\begin{array}{l}\text { Poultry } \\
\text { farm } \\
\text { worker }\end{array}$ & $\begin{array}{l}\text { Slaughterhouse } \\
\text { worker }\end{array}$ & $\begin{array}{l}\text { Poultry } \\
\text { farm } \\
\text { worker }\end{array}$ & $\begin{array}{c}\text { Slaughterhouse } \\
\text { worker }\end{array}$ & $\begin{array}{l}\text { Poultry } \\
\text { farm } \\
\text { worker }\end{array}$ & $\begin{array}{c}\text { Slaughterhouse } \\
\text { worker }\end{array}$ \\
\hline & Plain & 00 & 00 & 02 & 00 & 25 & 02 \\
\hline & Field & 02 & 01 & 00 & 00 & 21 & 01 \\
\hline & Market & 00 & 06 & 00 & 03 & 00 & 45 \\
\hline \multicolumn{8}{|c|}{$\begin{array}{r}\chi 2 \text { statistics for Poultry farm Workers }=4.054, \mathrm{df}=2, \mathrm{P} \text { value }=0.132, \text { Non-Significant as } P>0.05 \\
\chi 2 \text { statistics for Slaughterhouse Workers }=3.222, \mathrm{df}=4, \mathrm{P} \text { value }=0.521, \text { Non-Significant as } P>0.05 \\
\text { For poultry farm worker } 4 \text { cells }(77.8 \%) \text { have expected count }<5, \text { so Fisher's exact test }=3.296, P \\
\text { value }=0.167, \text { Non-Significant as } P>0.05 \\
\text { For slaughterhouse worker } 7 \text { cells }(66.7 \%) \text { have expected count }<5 \text {, so Fisher's exact test }=4.444, P \\
\text { value }=0.541, \text { Non-Significant as } P>0.05\end{array}$} \\
\hline \multicolumn{8}{|c|}{$\begin{array}{l}\text { Have many } \\
\text { rat/mice at home? }\end{array}$} \\
\hline Ye & & 02 & 00 & 00 & 00 & 21 & 06 \\
\hline No & & 00 & 07 & 02 & 03 & 25 & 42 \\
\hline \multicolumn{8}{|c|}{$\begin{array}{l}\chi 2 \text { statistics for Poultry farm Workers }=4.054, \mathrm{df}=2, \mathrm{P} \text { value }=0.132, \text { Non-Significant as } \mathrm{P}>0.05 \\
\chi 2 \text { statistics for Slaughterhouse Workers }=1.394, \mathrm{df}=2, \mathrm{P} \text { value }=0.498 \text {, Non-Significant as } \mathrm{P}>0.05 \\
\text { For poultry farm worker } 4 \text { cells }(66.7 \%) \text { have expected count }<5 \text {, so Fisher's exact test }=3.296, \mathrm{P} \text { value }\end{array}$} \\
\hline
\end{tabular}


$=0.167$, Non-Significant as $\mathrm{P}>0.05$

For slaughterhouse worker 4 cells $(66.7 \%)$ have expected count $<5$, so Fisher's exact test $=0.612, \mathrm{P}$ value $=1.00$, Non-Significant as $\mathrm{P}>0.05$

Have livestock in

home?

\begin{tabular}{|l|l|l|l|l|l|l|}
\hline Yes & 01 & 01 & 00 & 00 & 01 & 00 \\
\hline No & 01 & 06 & 02 & 03 & 45 & 48 \\
\hline
\end{tabular}

$\chi 2$ statistics for Poultry farm Workers $=11.50, \mathrm{df}=2, \mathrm{P}$ value $=0.003$, Significant as $\mathrm{P}<0.05$

$\chi 2$ statistics for Slaughterhouse Workers $=7.414, \mathrm{df}=2, \mathrm{P}$ value $=0.025$, Non-Significant as $\mathrm{P}>0.05$

$\chi 2$ statistics for both Work groups $=13.765, \mathrm{df}=2, \mathrm{P}$ value $=0.001$, Significant as $\mathrm{P}<0.05$

For poultry farm worker 5 cells $(83.3 \%)$ have expected count $<5$, so Fisher's exact test $=6.719$, $\mathrm{P}$ value $=0.155$, Non-Significant as $\mathrm{P}>0.05$

For slaughterhouse worker 4 cells $(66.7 \%)$ have expected count $<5$, so Fisher's exact test $=5.854, \mathrm{P}$ value $=0.172$, Non-Significant as $\mathrm{P}>0.05$

\section{Have many \\ rat/mice at work \\ place?}

\begin{tabular}{|l|l|l|l|l|l|l|}
\hline Yes & 02 & 07 & 02 & 03 & 46 & 48 \\
\hline No & 00 & 00 & 00 & 00 & 00 & 00 \\
\hline
\end{tabular}

Have a live stock

at work place?

\begin{tabular}{|l|l|l|l|l|l|l|}
\hline Yes & 02 & 07 & 01 & 03 & 07 & 46 \\
\hline No & 00 & 00 & 01 & 00 & 39 & 02 \\
\hline
\end{tabular}

$\chi 2$ statistics for Poultry farm Workers $=9.783, \mathrm{df}=2, \mathrm{P}$ value $=\mathbf{0 . 0 0 8}$, Non-significant as $P>0.05$ $\chi 2$ statistics for Slaughterhouse Workers $=0.432, \mathrm{df}=2, \mathrm{P}$ value $=0.806$, Non-Significant as $P>0.05$

For poultry farm worker 4 cells $(66.7 \%)$ have expected count $<5$, so Fisher's exact test $=7.814, P$ value $=0.022$, Significant as $P<0.05$

For slaughterhouse worker 4 cells $(66.7 \%)$ have expected count $<5$, so Fisher's exact test $=1.038, P$ value $=1.00$, Non-Significant as $P>0.05$

Presence of rat/

mice in drain of

work place?

\begin{tabular}{|l|l|l|l|l|l|l|}
\hline Yes & 02 & 04 & 00 & 01 & 11 & 15 \\
\hline No & 00 & 03 & 02 & 02 & 35 & 33 \\
\hline
\end{tabular}

$\chi 2$ statistics for Poultry farm Workers $=0.708, \mathrm{df}=2, \mathrm{P}$ value $=0.702$, Non-significant as $P>0.05$ $\chi 2$ statistics for Slaughterhouse Workers $=0.212, \mathrm{df}=2, \mathrm{P}$ value $=0.899$, Non-Significant as $P>0.05$

For poultry farm worker 4 cells $(66.7 \%)$ have expected count $<5$, so Fisher's exact test $=0.503, P$ value $=1.00$, Non-Significant as $P>0.05$

For slaughterhouse worker 4 cells $(66.7 \%)$ have expected count $<5$, so Fisher's exact test $=2.003, P$ value $=1.00$, Non-Significant as $P>0.05$ 
Table.4 Comparisons of Leptospira spp. IgG ELISA result with Behavioural risk factor

\begin{tabular}{|c|c|c|c|c|c|c|c|}
\hline \multirow{2}{*}{\multicolumn{2}{|c|}{ Variable }} & \multicolumn{6}{|c|}{ Leptospira spp.IgG ELISA Result } \\
\hline & & \multicolumn{2}{|c|}{ Positive n $(\%)$} & \multicolumn{2}{|c|}{ Equivocal n (\%) } & \multicolumn{2}{|c|}{ Negative $n(\%)$} \\
\hline \multicolumn{2}{|c|}{$\begin{array}{l}\text { Walk in mud/ } \\
\text { dung? }\end{array}$} & $\begin{array}{l}\text { Poultry } \\
\text { Farm } \\
\text { worker }\end{array}$ & $\begin{array}{l}\text { Slaughterhouse } \\
\text { worker }\end{array}$ & $\begin{array}{l}\text { Poultry } \\
\text { farm } \\
\text { worker }\end{array}$ & $\begin{array}{l}\text { Slaughterhouse } \\
\text { worker }\end{array}$ & $\begin{array}{l}\text { Poultry } \\
\text { farm } \\
\text { worker }\end{array}$ & $\begin{array}{l}\text { Slaughterhouse } \\
\text { worker }\end{array}$ \\
\hline \multicolumn{2}{|l|}{ Yes } & 02 & 07 & 00 & 03 & 05 & 48 \\
\hline \multicolumn{2}{|l|}{ No } & 00 & 00 & 02 & 00 & 41 & 00 \\
\hline \multicolumn{8}{|c|}{$\begin{array}{r}\chi^{2} \text { for } \mathrm{Pc} \\
\chi^{2} \text { statistic } \\
\text { For poultry far }\end{array}$} \\
\hline \multicolumn{8}{|c|}{$\begin{array}{l}\text { Total time } \\
\text { spending / day (in } \\
\text { working) }\end{array}$} \\
\hline \multicolumn{2}{|c|}{5 hrs } & 00 & 06 & 00 & 03 & 00 & 41 \\
\hline \multirow{2}{*}{\multicolumn{2}{|c|}{$\begin{array}{l}6 \text { to } 7 \text { hrs } \\
8 \text { hrs and } \\
\text { more }\end{array}$}} & 00 & 01 & 00 & 00 & 00 & 07 \\
\hline & & 02 & 00 & 02 & 00 & 46 & 00 \\
\hline & \multicolumn{7}{|c|}{$\begin{array}{c}\chi 2 \text { statistics for Poultry farm Workers was not compared because of constant response } \\
\chi 2 \text { statistics for Slaughterhouse Workers }=0.507, \mathrm{df}=2, \mathrm{P} \text { value }=0.776 \text {, Non-Significant as P }>0.05 \\
\text { For slaughterhouse worker } 4 \text { cells }(66.7 \%) \text { have expected count }<5 \text {, so Fisher's exact test }=0.355, \mathrm{P} \\
\text { value }=1.00 \text {, Non-Significant as } \mathrm{P}>0.05\end{array}$} \\
\hline \multicolumn{2}{|c|}{$\begin{array}{l}\text { Have } \\
\text { wound in } \\
\text { present? }\end{array}$} & & & & & & \\
\hline \multicolumn{2}{|l|}{ Yes } & 00 & 03 & 00 & 01 & 00 & 02 \\
\hline \multicolumn{2}{|l|}{ No } & 02 & 04 & 02 & 02 & 46 & 46 \\
\hline \multicolumn{8}{|c|}{$\begin{array}{c}\chi 2 \text { statistics for Poultry farm Workers was not compared because of constant response } \\
\chi 2 \text { statistics for Slaughterhouse Workers }=11.663, \mathrm{df}=2, \mathrm{P} \text { value }=0.003, \text { Significant as } \mathrm{P}<0.05 \\
\chi 2 \text { statistics for both Work groups }=17.329, \mathrm{df}=2, \mathrm{P} \text { value }=0.000, \text { Significant as } \mathrm{P}<0.05 \\
\text { For slaughterhouse worker } 4 \text { cells }(66.7 \%) \text { have expected count }<5, \text { so Fisher's exact test }=9.893, \mathrm{P} \\
\text { value }=0.007, \text { Significant as } \mathrm{P}<0.05\end{array}$} \\
\hline \multicolumn{8}{|c|}{$\begin{array}{l}\text { Wound in } \\
\text { past? }\end{array}$} \\
\hline \multicolumn{2}{|c|}{ Yes } & 00 & 07 & 00 & 03 & 00 & 47 \\
\hline \multicolumn{2}{|l|}{ No } & 02 & 00 & 02 & 00 & 46 & 01 \\
\hline \multicolumn{8}{|c|}{$\begin{array}{c}\chi 2 \text { statistics for Poultry farm Workers was not compared because of constant response } \\
\chi 2 \text { statistics for Slaughterhouse Workers }=0.212, \mathrm{df}=2, \mathrm{P} \text { value }=0.899, \text { Non-Significant as } P>0.05 \\
\text { For slaughterhouse worker } 4 \text { cells }(66.7 \%) \text { have expected count }<5 \text {, so Fisher's exact test }=2.003, P \\
\text { value }=1.00 \text {, Non-Significant as } P>0.05\end{array}$} \\
\hline \multicolumn{2}{|c|}{$\begin{array}{l}\text { Contact with } \\
\text { animal? }\end{array}$} & & & & & & \\
\hline \multicolumn{2}{|l|}{ Dog } & 00 & 00 & 00 & 00 & 03 & 00 \\
\hline
\end{tabular}




\begin{tabular}{|c|c|c|c|c|c|c|}
\hline Livestock & 00 & 07 & 00 & 03 & 05 & 48 \\
\hline $\begin{array}{l}\text { Dog \& Livestock } \\
\text { both }\end{array}$ & 02 & 00 & 02 & 00 & 38 & 00 \\
\hline \multicolumn{7}{|c|}{$\begin{array}{c}\chi 2 \text { for Poultry farm Workers }=2.446, \mathrm{df}=6, \mathrm{P} \text { value }=0.874, \text { Non-Significant as } \mathrm{P}>0.05 \\
\chi 2 \text { statistics for Slaughterhouse Workers was not compared because of constant response } \\
\text { For poultry farm worker } 10 \text { cells }(83.3 \%) \text { have expected count }<5, \text { so Fisher's exact test }=3.121, P \\
\text { value }=1.00, \text { Non-Significant as } P>0.05\end{array}$} \\
\hline \multicolumn{7}{|l|}{$\begin{array}{l}\text { Always wash } \\
\text { hands } \\
\text { immediately after } \\
\text { contact with } \\
\text { animal? }\end{array}$} \\
\hline Yes & 02 & 00 & 02 & 01 & 46 & 07 \\
\hline No & 00 & 07 & 00 & 02 & 00 & 41 \\
\hline \multicolumn{7}{|c|}{$\begin{array}{c}\chi^{2} \text { statistics for Poultry farm Workers was not compared because of constant response } \\
\chi 2 \text { statistics for Slaughterhouse Workers }=2.109, \text { df }=2, P \text { value }=0.348, \text { Non-Significant as } P>0.05 \\
\text { For slaughterhouse worker } 3 \text { cells }(66.7 \%) \text { have expected count }<5 \text {, so Fisher's exact test }=2.049, P \\
\text { value }=0.265, \text { Non-Significant as } P>0.05\end{array}$} \\
\hline \multicolumn{7}{|l|}{$\begin{array}{l}\text { Wearing shoes } \\
\text { while working? }\end{array}$} \\
\hline Yes & 02 & 02 & 02 & 01 & 45 & 15 \\
\hline No & 00 & 05 & 00 & 02 & 01 & 33 \\
\hline \multicolumn{7}{|c|}{$\begin{array}{c}\chi 2 \text { statistics for Poultry farm Workers }=0.089, \mathrm{df}=2, \mathrm{P} \text { value }=0.957, \text { Non-significant as } P>0.05 \\
\chi 2 \text { statistics for Slaughterhouse Workers }=0.028, \mathrm{df}=2, \mathrm{P} \text { value }=0.986, \text { Non-Significant as } P>0.05 \\
\text { For slaughterhouse worker } 4 \text { cells }(66.7 \%) \text { have expected count }<5, \text { so Fisher's exact test }=0.306, P \\
\text { value }=1.00, \text { Non-Significant as } P>0.05\end{array}$} \\
\hline \multicolumn{7}{|l|}{$\begin{array}{l}\text { Washing foot } \\
\text { immediately after } \\
\text { becoming wet } \\
\text { from dirty water? }\end{array}$} \\
\hline Yes & 00 & 04 & 00 & 02 & 02 & 29 \\
\hline No & 02 & 03 & 02 & 01 & 44 & 19 \\
\hline \multicolumn{7}{|c|}{$\begin{array}{r}\chi 2 \text { statistics for Poultry farm Workers }=0.181, \mathrm{df}=2, \mathrm{P} \text { value }=0.913, \text { Non-significant as } P>0.05 \\
\chi 2 \text { statistics for Slaughterhouse Workers }=0.080, \mathrm{df}=2, \mathrm{P} \text { value }=0.961, \text { Non-Significant as } P>0.05 \\
\text { For poultry farm worker } 5 \text { cells }(83.3 \%) \text { have expected count }<5 \text {, so Fisher's exact test }=1.878, P \\
\text { value }=1.00, \text { Non-Significant as } P>0.05 \\
\text { For slaughterhouse worker } 4 \text { cells }(66.7 \%) \text { have expected count }<5 \text {, so Fisher's exact test }=0.294, P \\
\text { value }=1.00, \text { Non-Significant as } P>0.05\end{array}$} \\
\hline
\end{tabular}

While comparing Human Leptospira spp. IgG ELISA result with behavioral risk factor we reported all sero-positive poultry farm workers had history of walking in mud/dung, also they did not wash their feet immediately after becoming wet with dirty water. They spent 8 hours and more per day at their workplaces and had history of contacts with dog and livestock. Where in slaughterhouse workers, we reported all sero-positive slaughterhouse workers walked in mud/dung during occupational practice and did not wash 
their hands after completion of the respective task or after contact with animals. In the present study it was found that the majority of sero-positive slaughterhouse workers spent 5 hours/day at their workplaces. Three (3) seropositive slaughterhouse workers out of seven (7) had wound in the present. All seropositive slaughterhouse workers had wound in the past and had contact with livestock. The majority of sero-positive slaughterhouse workers did not wear shoes and did not wash their feet immediately after becoming wet with dirty water.

To date very few studies were conducted in this high-risk group and this study is probably first in India in the context of poultry farm workers and Leptospira spp. infection with the best of our knowledge. The current study showed that the prevalence of Leptospira spp. among the poultry farm workers of Navsari district was $4 \%(n=50)$. However, Bernkopf (1948) [6] reported an experimental infection of chickens by feeding them a fluid culture of Leptospira with a pipette, whereas, Gillespie et al., (1953) [6] observed positive agglutination-lysis tests for Leptospira pomona in chickens on a ranch where active bovine Leptospirosis was diagnosed, Chalquest et al., (1957) [6] stated that the exact role poultry may play in this disease is unknown. Everard et al., (1985) [7] found 11 $\%$ positive sera in 175 chickens of Grenada and $11 \%$ positive sera in 144 chickens of Trinidad. Beran (1994) [8] stated that, although there are few reports of isolation of Leptospires from wildfowl, it is generally accepted that clinical disease does not exists. However, we haven't studied poultry as a source of infection as most of the available literature suggests that poultry is known to be resistant to Leptospirosis. But Villafane et al., (2004) [12] stated, that, foodstuffs available at poultry farms is the source of attraction to wild rodents, resulting into the possibility of getting favorable condition at a poultry farm, especially unhygienic conditions, posing a risk of contracting Leptospirosisto poultry farm workers. Rodents roaming around poultry farm may acts as reservoirs was proven by the study conducted by Balamurugan et al., (2013) [13]. They reported Leptospira spp. isolate in heart blood of rat trapped at a poultry farm. So we studied animals and rats present at poultry farms, however, we did not report any Leptospira spp. Indirect ELISA positive rat. But, we reported one Indirect ELISA positive cattle at poultry farm.

Although slaughterhouse workers had known risk of exposure to many zoonotic infections and very few studies were conducted pertaining to this occupation in the Gujarat state. The study showed that the prevalence of Leptospira spp. among the slaughterhouse workers was $12.06 \% \quad(\mathrm{n}=58)$. Although, Sarwankar et al., (2017) [3] reported prevalence in farm workers $(56 \%)$, farmers $25 \%$ and others $19 \%$, Whereas, Sharma et al., (2006) [11] reported a $30 \%(\mathrm{n}=20)$ seroprevalence of Leptospirosis among slaughterhouse workers of Andaman Island, by considering IgM- and/or IgG-ELISA positive sera as cases of Leptospirosis. This high prevalence may be due to less samples size. Furthermore, Cook et al., (2016) [12] reported $13.4 \%$ sero-prevalence of IgMantibodies to Leptospira spp. in slaughterhouse workers of Western Kenya. Alvarado-Esquivel et al., (2016) [13] reported $17.7 \%$ of Anti-Leptospira IgG antibodies in meat workers of Northern Mexico. Benschop et al., (2009) [14] reported 9.5\% seroprevalence of Leptospirosis by Microscopic Agglutination Test in slaughterhouse workers of New Zealand slaughterhouse, which was less than our results.

In the present study we have not find any strong correlation between Leptospira spp. IgG ELISA result and sex of worker, as one 
of sero-positive poultry farm workers was male and one was female. But there are some reports who claim that's male are more susceptible to Leptospirosis such as Jansen et al., (2007) [15] reported that male has higher severity of clinical Leptospirosis compare to female. Puca et al., [16] reported a higher prevalence of Leptospirosis in middle aged men. We found that all sero-positive poultry farm workers were above 25 years of age, but Gancheva et al., (2016) [17] described age as a prognostic factor in Leptospirosis and reported that age over 45 years is associated with a severe course of Leptospirosis, has higher risk for death and requires early intensive treatment. In present study, one of the sero-positive poultry farm worker was more than 45 years. Whereas, education-wise one of the sero-positive poultry farm workers was illiterate and one had higher secondary education, although lack of awareness leads to reduce the perceived threat of the disease, which could minimize taking preventive measures, Agampodi et.al, 2010 [18]. When we consider the work experience all the seropositive poultry farm worker had work experience of 5-19 years. Whereas, in slaughterhouse workers all the Leptospira spp. IgG ELISA positive slaughterhouse workers were male, as this industry has traditionally been a male-dominated occupation and remains significantly so as demonstrated. Age group-wise majority of sero-positive slaughterhouse workers were more than 25 years of age. What was noteworthy was that $67 \%$ of the slaughterhouse workers were younger than 45 years, with many productive years ahead of them. Education-wise majority of seropositive slaughterhouse workers had higher secondary education. But lack of awareness about the zoonotic disease will make them prone to Leptospirosis. Work experience-wise majority of sero- positive slaughterhouse workers had work experience of 20 years and above, they would likely have previous sub- clinical infections. For both the workgroup chi-square test was significant $(\mathrm{p}<0.05)$ for work experience and Leptospira spp. IgG ELISA results.

Environment suitability for the survival of organism appears to be a crucial factor in maintaining and transmission of Leptospirosis to humans. In this study all Leptospira spp. IgG positive poultry farm workers were living in field areas either in poultry farm itself or in agriculture farm. Both these areas were full of surrounding rodents which serves as a major risk factor for them. The study demonstrated that the environmental risk factors for Leptospirosis are prevalent among the poultry farm and slaughterhouse workers. Since sustainable food production is a major concern, finding an alternative to the industrial meat supply chain is almost impossible [19]. At the time of inspection, the presence of Livestock animals and stray dogs were also observed either on or near some of the premises of poultry farms. These animals may expose humans to several serovars of Leptospira spp. So it is important to vaccinate them against Leptospira spp. The presence fodder, open drainage, poultry farm waste, stored water left unprotected overnight and the presence of rodents increases the risk of possible contamination with their urine, serving as a source of infection for both man and animals. Surprisingly it is observed that the majority of slaughterhouse workers were unaware of the meaning of "Leptospirosis." and they don't know about rodents being the carrier of Leptospirosis and can transmit the infection. This highlights the need for continuous education and training in the study population about food hygiene. Furthermore, we reported that workers were constantly exposed to feces, blood, and other liquid waste from slaughtered animals and not using appropriate personal protective equipment (PPE). But, they regularly washing down of the floors and walls of the premises, this will 
remove contaminants and reduce the risk of cross-contamination of products and infection to workers. Periodical screening of these workers for different zoonotic diseases including Leptospirosis can play an important role for early diagnosis and intervention to prevent such infection among these workers. The presence of slaughterhouse waste which attracts rodents may increase the risk of possible contamination, with their urine serving as a source of infection for workers and animals present at workplace. If the blood of infected slaughtered animals present at slaughter houses, if not properly disposed of it may become a source of infection for the workers and even a wider environment becomes at risk in the event of rainfall and local flooding. Moreover, the absence of personal protective equipment (PPE), or their inconsistent usage, would undoubtedly expose the workers to possible infection resulting in increased risk to zoonotic diseases [19]. Sugunan et al., (2009) [20] reported that the presence of cattle in the house, drinking stream water, contact with garbage, walking barefoot, and standing in water while working were identified as significant risk factors associated with Leptospirosis.

Vanasco et al., (2008) [21] stated that age >30 years, occupation in a rural setting associated with animals, contact with contaminated surface water, and contact with floods were significantly associated with Leptospirosis. In the present study, while comparing Leptospiraspp IgG ELISA results with behavioral risk factors we reported two of sero-positive poultry farm workers were walking in mud/dung while working on poultry farms $(\mathrm{p}<0.05)$. All the sero-positive poultry farm workers spent at least 8 hours and more at the workplace, had contact with Dogs and Livestock and did not wash foot immediately after becoming wet with dirty water. Whereas, in slaughterhouse workers it was observed that all the Leptospira spp. IgG
ELISA positive slaughterhouse workers were walking in mud/dung, were spent at least 5 hours per day at the slaughterhouse and did not wash hands with soap after completion of the respective task which was found the risk factors for Leptospirosis. In addition with these one interesting fact was observed that three of the sero-positive slaughterhouse workers had wound in present $(\mathrm{p}<0.05)$. Cook et al., (2016) [12]already reported some of these risk factors like having wounds, smoking, eating at work and cleaning the offal. All the sero-positive slaughterhouse workers had wound in the past, which is common in this traditional occupation. But it shows that this working group requires education regarding first-aid treatments and wound hygiene.

The negligence of slaughterhouse workers about the preventive measures such as proper treatment of wounds, not washing hands with soap and water after completion of task will make them prone to various infectious diseases including Leptospirosis. Brockmann et al., (2016) [22] reported that contact with pet rats, guinea pigs, cattle and poultry or livestock as well as forestry worker occupation were identified as important exposure factors. In the present study we observe that all sero- positive slaughterhouse workers have contact with livestock and lack of hand washing practice after animal contacts. The majority of sero positive slaughterhouse workers did not wear shoes while slaughtering animals, they said that it's difficult to perform slaughter wearing shoe.

Surprisingly it was observed that rats present on poultry farms were found negative for Leptospira spp. indirect ELISA. Whereas, one of cattle present at poultry farm was found positive for Leptospira spp. indirect ELISA. Keenan J et al., (2009) [23] reported a $27 \%$ sero-prevalence in rats and Sharma et al., (2014) [24] reported Leptospiral antibodies in 
$34 \%$ of Cattles and $29 \%$ of Goats slaughter in different Government-owned slaughterhouse of Andaman Island. In the present investigation we reported one Leptospira spp. indirect ELISA positive buffalo at the slaughterhouse. We tested a total of 83 serum samples of different species of animals among them we reported only two $(2.40 \%)$ were positive for Leptospira spp. indirect ELISA. In poultry farms we tested 28 serum samples and reported only one (3.57\%) positive. Whereas in slaughterhouses we tested 55 serum samples and reported only one (1.81\%) positive. This shows the presence of Leptospiral antibodies in carrier and reservoir animals present at their workplaces.

In conclusions the prevalence of Leptospira spp. among poultry farm workers was $4 \%$ $(\mathrm{n}=50)$ and in slaughterhouse workers was $12.06 \%(\mathrm{n}=58)$, so we can say that these working group had a risk of getting infected with Leptospira spp. We reported long working hours, unhygienic practices like not wearing PPE, lack of hand washing practice after completion of respective task as well as after animal contacts, no washing of legs after becoming wet with dirty water, walking in mud or dung, not wearing a shoe, having wound in present/past, living infield area, presence of rats and livestock at the workplace as well as at home will increase risk of getting infected with Leptospira spp.in poultry farm and slaughterhouse workers. We found Leptospiral antibodies in $3.57 \%$ and $1.81 \%$ of carrier and reservoir animals present at poultry farms and slaughtered at slaughterhouses respectively.

\section{Limitation}

In this study, we did Leptospira spp.IgG ELISA, which might give positive result years after infection. So we cannot determine that this IgG positive result is because of recent infection or past infection. So it requires elaborate research with more than one sensitive and specific parameter to find exact time of infection in this work group and to corelate with their routine activities.

\section{Author Contributions}

Conceptualization, Yogendra Mevada, Rajendra Palkhade and Utpal Kumar Chattopadhyay; Data curation, Yogendra Mevada, Subhrajit Sarkar and Jitendra Parmar; Formal analysis, Yogendra Mevada, Rajendra Palkhade, utpal Kumar Chattopadhyay, Amit Banik, Sayan Bhattacharyya, Subhrajit Sarkar and Nisha Gupta; Funding acquisition, Yogendra Mevada, Rajendra Palkhade and Utpal Kumar Chattopadhyay; Investigation, Yogendra Mevada, Rajendra palkhade,utpal Kumar Chattopadhyay, Amit Kanani, Subhrajit Sarkar, Nisha Gupta and Jitendra Parmar; Methodology, Yogendra Mevada, Rajendra Palkhade, Utpal Kumar Chattopadhyay; Project administration, Rajendra Palkhade, Utpal Kumar Chattopadhyay and Atul Raj; Resources, Yogendra Mevada, Rajendra Palkhade, Utpal KumaR Chattopadhyay, Amit Kanani, Atul Raj, Amit Banik, Sayan Bhattacharyya, Subhrajit Sarkar, Nisha Gupta and Jitendra Parmar; Software, YogendrA Mevada and Subhrajit Sarkar; Supervision, Rajendra Palkhade and Utpal Kumar Chattopadhyay; Visualization, Yogendra Mevada, Rajendra Palkhade, Utpal kumar Chattopadhyay and Amit Kanani; Writing original draft, Yogendra Mevada; Writing review \& editing, Yogendra Mevada, Rajendra Palkhade, Utpal Kumar Chattopadhyay, Amit Kanani, Amit Banik, Sayan Bhattacharyya, Nisha Gupta and Jitendra Parmar.

\section{Funding}

We are thankful to The Director, AIIH \& PH, Kolkata, for financially supporting Human ELISA analyses. 


\section{Acknowledgment}

We acknowledge support and assistance received from ICMR-NIOH, Ahmedabad; AIIH \& PH, MOH \& FW, Kolkata; Dept. of Animal Husbandry, Govt. of Gujarat; AMC, Ahmedabad; SMC, Surat, and Genomix Molecular Diagnostics Private Limited, Hyderabad.

\section{Conflicts of Interest}

There are no conflicts of interest.

\section{References}

1. Shivakumar, S. Leptospirosis - Indian Data 2013. Research Gate, 2014.

2. Patel, J. M.; Vihol, P. D.; Dabas, V. S.; Prasad, M. C.; Patel, J. H.; Chaudhari, C. F.; Patel, N. B.\& Patel, K. M.Seroepidemiological study of leptospirosis in buffaloes of south Gujarat, India. Buffalo Bulletin, 2016, 35 (3), 383-388.

3. Ashvika, S.; Summaiya, M. Prevalence of Leptospirosis in various risk groups of South Gujarat, India. International Journal of Management and Applied Science (IJMAS). 2017, 5 (2), Spl. Iss-2, 53-55.

4. Gayatri, P.; Pol, S.; Bharadwaj, R. Prevalence of Leptospirosis among paddy field workers in Pune, Western India. Int J Cur Res Rev.2017, 9 (5), 22-25.

5. Singh $\mathrm{T}$ et al. Socio-economic status scales updated for 2017. Int J Res Med Sci. 2017, Jul;5(7):3264-3267.

6. Chalquest, R. R. Experimental infection of Birds with Leptospira pomonapresent in Bovine urine and preliminary studies on their role in transmission of Leptospirosis. Poultry science. 1957, 36(1),110-113.

7. Everard, C.O.R.; Fraser-Chanpong, G.M.; James, A.C.; Butcher, L.V. Serological studies on Leptospirosis in livestock and chickens from Grenada and Trinidad. Trans. $R$. Soc. Trop. Med. Hyg. 1985. 79, 859-864.

8. Michael, T.; Marshall, R. B. (1994). Leptospirosis. Handbook of Zoonoses, Second Edition, Section A: Bacterial, Rickettsial, Chlamydial, and MycoticZoonoses. George W. Beran, Second edition, CRC Press. Inc: Boca Raton, Florida, USA, 1994; Section A.

9. Villafañeet al. Assessment of the risks of rats (Rattusnorvegicus) and opossums (Didelphisalbiventris) in different poultry-rearing areas in argentina. Brazilian Journal of Microbiology. 2004, 35, 359-363.

10. Balamurugan, V.; Gangadhar, N. L.; Mohandoss, N.; Thirumalesh, S. R. A.; Dhar,M.; Shome, R. \&Rahman, H. Characterization of leptospira isolates from animals and humans: phylogenetic analysis identifies the prevalence of intermediate species in India. SpringerPlus. 2013,2, 362.

11. Sharma, S.; Vijayachari, P.; Sugunan, A. P.; Natarajaseenivasan, K.; Sehgal S. C. Seroprevalence of Leptospirosis among high-risk population of Andaman Islands, India Am. J. Trop. Med. Hyg.2006,74(2), 278-283.

12. Cook, E. A. J.; de Glanville, W. A.; Thomas, L. F.; Kariuki, S.;Bronsvoort, B. M. de C.; Fèvre, E. M. Risk factors for leptospirosis seropositivity in slaughterhouse workers in western Kenya. Occupational and Environmental Medicine. 2017, 74(5), 357-365.

13. Alvarado-Esquivel $\mathrm{C}$, Hernandez-Tinoco $\mathrm{J}$, Sanchez-Anguiano LF, et al. High Seroprevalence of Leptospira Exposure in Meat Workers in Northern Mexico: A Case-Control Study. J Clin Med Res. 2016;8(3):231-236.

14. Benschop, J.; Heuer, C.; Jaros, P.; Collins-emerson, J.; Midwinter, A. \& 
Wilson P. Sero-prevalence of leptospirosis in workers at a New Zealand slaughterhouse. $\quad N \quad Z \quad M e d \quad J$. 122(1307).2009, 39-47.

15. Jansen, A.; Stark, K.; Schneider, T. \&Scho“ neberg, I. Sex Differences in Clinical Leptospirosis in Germany: 19972005. Clinical Infectious Diseases.2007, 44, e69-e72.

16. Pucaet al. The role of gender in human leptospirosis.J Infect

DevCtries.2018,12(3):150-155

17. Gancheva, G. I. Age as Prognostic Factor in Leptospirosis. Annals of Infectious Disease and Epidemiology.2016, 1(2), 1 6.

18. Agampodi, S. B., Agampodi, T. C., Thalagala, E., Perera, S., Chandraratne, S., \& Fernando, S. Do People Know Adequately about Leptospirosis? A Knowledge Assessment Survey in Postoutbreak Situation in Sri Lanka. International journal of preventive medicine.2010, 1(3), 158-163.

19. Brown, P. D.; Mckenzie, M.; Pinnock, M. \&Mcgrowder, D. Environmental risk factors associated with leptospirosis among butchers and their associates in Jamaica. Int J Occup Environ Med. 2011,
2(1), 47-57.

20. Sugunan, A. P.; Vijayachari, P.; Sharma, S. et al. Risk factors associated with leptospirosis during an outbreak in Middle Andaman, India. Indian J Med Res.2009, 130(1), 67-73.

21. Vanascoa, N. B.; Schmelinga, M. F.; Lottersberger, J.; Costac, F. et.al. Clinical characteristics and risk factors of human leptospirosis in Argentina (1999-2005). ActaTropica.2008, 107, 255-258.

22. Brockmann, S. O.; Ulrich, L.; Piechotowski, I.; Wagner-Wiening, C.; Nöckler, K.; Mayer- Scholl, A.; Eichner, M. Risk factors for human Leptospira seropositivity in South Germany. SpringerPlus. 2016, 5(1), 1796.

23. Keenan. J.; Sharma, R.; Dicker, R.; Rayner, J.; Stone D. Seroprevalence of Leptospira in rattusnorvegicus in Grenada, West Indies. West Indian Med J.2009, 58(2), 114-7.

24. Sharma, S.; Vijayachari, P.; Sugunan, A. P.; Roy, S.; Kalimuthusamy, N. Seroprevalence and Carrier Status for Leptospirosis in Cattle and Goats in Andaman Island, India. Journal of Veterinary Science \& Technology.2014, 05. 10.4172/2157- 7579.1000205.

\section{How to cite this article:}

Yogendra Mevada, Rajendra Palkhade, Utpal Kumar Chattopadhyay, Amit Kanani, Atul Raj, Amit Banik, Sayan Bhattacharyya, Subhrajit Sarkar, Nisha Gupta and Jitendra Parmar. 2020. Serological Survey of Leptospirosis among Poultry Farm and Slaughterhouse Workers and its Co-relation with the Risk Factors. Int.J.Curr.Microbiol.App.Sci. 9(09): 3556-3571. doi: https://doi.org/10.20546/ijcmas.2020.909.441 